\title{
Primary Cough Headache
}

National Cancer Institute

\section{Source}

National Cancer Institute. Primary Cough Headache. NCI Thesaurus. Code 6117078.

Headache triggered by coughing or straining in the absence of an intracranial disorder. 